\title{
Regulation and function of capicua in mammals
}

\author{
Yoontae Lee Let, $^{1,2}$
}

\begin{abstract}
Capicua $(\mathrm{CIC})$ is an evolutionarily conserved transcription factor. CIC contains a high-mobility group (HMG) box that recognizes specific DNA sequences to regulate the expression of various target genes. CIC was originally identified in Drosophila melanogaster as a transcriptional repressor that suppresses the receptor tyrosine kinase signaling pathway. This molecule controls normal organ growth and tissue patterning as well as embryogenesis in Drosophila. Recent studies have also demonstrated its extensive functions in mammals. For example, CIC regulates several developmental and physiological processes, including lung development, abdominal wall closure during embryogenesis, brain development and function, neural stem cell homeostasis, T cell differentiation, and enterohepatic circulation of bile acids. CIC is also associated with the progression of various types of cancer and neurodegeneration in spinocerebellar ataxia type-1, systemic autoimmunity, and liver injury. In this review, I provide a broad overview of our current understanding of the regulation and functions of CIC in mammals and discuss future research directions.
\end{abstract}

\section{Introduction}

In 2000, the capicua (cic) gene was first identified in Drosophila melanogaster as a transcriptional repressor involved in the regulation of embryogenesis ${ }^{1}$. Casanova and colleagues performed a P-element screen to identify genes required for anteroposterior patterning in Drosophila ${ }^{1}$. These researchers found that a mutant embryonic phenotype characterized by a lack of abdominal segmentation but maintenance of head and tail structures was caused by a mutation in capicua (thus explaining the gene name, derived from the Catalan term meaning "head-and-tail") ${ }^{1}$. Cic is required for organ growth and tissue patterning as well as anteroposterior and dorsoventral formation during embryogenesis in Drosophila ${ }^{1-9}$. Cic represses the expression of genes downstream of receptor tyrosine kinases (RTKs), including Torso and epidermal growth factor receptor (EGFR) ${ }^{1,8}$. Therefore, Cic functions as a negative regulator of the RTK signaling pathway. Moreover, RTK signaling activation promotes the degradation and/or cytoplasmic translocation of $\mathrm{Cic}$ via phosphorylation, thereby inducing the expression of Cic target genes downstream of RTK pathways ${ }^{2,8,10}$.

Correspondence: Yoontae Lee (yoontael@postech.ac.kr)

'Department of Life Sciences, Pohang University of Science and Technology, Pohang, Gyeongbuk, Republic of Korea

${ }^{2}$ Yonsei University, Seoul, Republic of Korea
CIC is evolutionarily conserved from Caenorhabditis elegans to humans ${ }^{1,11,12}$. CIC exists as two isoforms, the short form (CIC-S) and the long form (CIC-L), which differ at their N-termini (Fig. 1a). CIC harbors two conserved domains, the high mobility group (HMG)-box and C1 domain (Fig. 1a), which cooperatively recognize specific octameric DNA sequences ${ }^{13}$. In mammals, CIC interacts with ataxin-1 (ATXN1), of which the polyglutamine (polyQ)-expanded form causes spinocerebellar ataxia type-1 (SCA1), a neurodegenerative disease ${ }^{14}$. CIC contributes to the pathogenesis of SCA1 in mice via interactions with mutant ATXN1 ${ }^{15,16}$. A fusion between $\mathrm{CIC}$ and a transcription activator domain of double homeobox 4 (DUX4) (CIC-DUX4 fusion protein) was identified in Ewing-like sarcoma cells ${ }^{17}$. CIC-DUX4 fusion proteins activate the expression of ETV1, ETV4, and ETV5, which encode oncogenic transcription factors $^{18}$, thereby promoting cancer progression ${ }^{17}$. Many studies have verified that $\mathrm{CIC}$ functions as a tumor suppressor in various types of cancer ${ }^{19-28}$. Endogenous functions of CIC have been elucidated by examinations of the phenotypes of $\mathrm{Cic}$ mutant mice. $\mathrm{CIC}$ deficiency results in defects in lung development, bile acid homeostasis, abdominal wall closure during embryogenesis, neuronal cell differentiation, brain development, and $\mathrm{T}$ cell subset differentiation ${ }^{25-27,29-34}$. In this review, I focus on the 


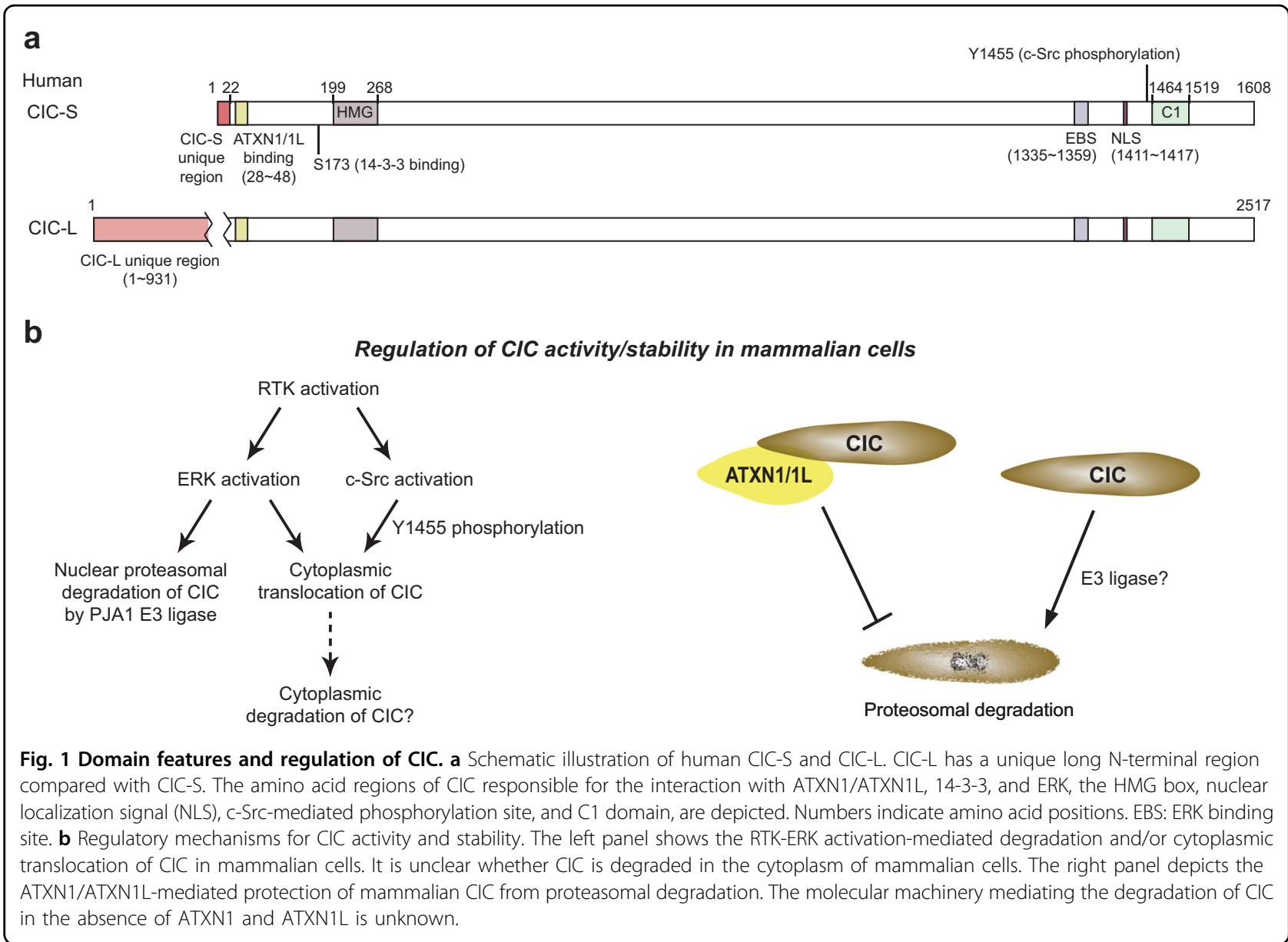

roles of CIC in mammals; in particular, I summarize recent studies of (1) its functions in diseases, including neurological diseases and cancer, (2) its functions in development, and (3) its underlying regulatory mechanisms in mammalian cells.

\section{CIC functions in diseases}

\section{Spinocerebellar ataxia type-1 (SCA1)}

SCA1 is one of nine polyQ disorders ${ }^{35,36}$. Expansion of the CAG repeat in ATXN1 results in a long polyQ tractcontaining mutant ATXN1, which is associated with cerebellar neurodegeneration primarily due to Purkinje cell death ${ }^{35}$. Phosphorylation at the S776 residue of ATXN1 is critical for the neurotoxicity of the polyQexpanded ATXN1 $1^{37,38}$. CIC binds with a high affinity to ATXN1 in human cells ${ }^{14}$. The CIC-ATXN1 complex is approximately $1.8 \mathrm{MDa}$ in size, irrespective of the polyQ expansion in ATXN $1^{14}$. The S776A mutation reduces the incorporation of ATXN1 into large CIC-ATXN1 complexes, implying that the interaction with $\mathrm{CIC}$ contributes to the neurotoxicity of the polyQ-expanded ATXN1 $1^{14}$. Fryer et al. experimentally proved that CIC facilitates the pathogenesis of SCA1 using a Cic-deficient SCA1 mouse model (Atxn1 ${ }^{154 Q}$; Cic-L $\left.{ }^{+/-}\right)$generated by crossing $154 \mathrm{Q}$ knock-in SCA1 (Atxn1 ${ }^{154 Q}$ ) mice with Cic hypomorphic $\left(\mathrm{Cic}^{-\mathrm{L}^{-1-}}\right)$ mice ${ }^{15}$. A partial loss of CIC expression substantially attenuated the pathological and behavioral abnormalities of the Atxn $1^{154 Q}$ mice ${ }^{15}$. Furthermore, the expression levels of some $\mathrm{CIC}$ target genes were downregulated in the cerebellum of the $\operatorname{Atxn} 1^{154 \mathrm{Q}}$ mice and were significantly rescued in the cerebellum of the Atxn1 ${ }^{154 Q} ; \mathrm{Cic}^{-L^{+/-}}$mice ${ }^{15}$. These findings suggest that the polyQ-expanded ATXN1 could enhance the transcriptional repressor activity of CIC for a subset of target genes, thereby contributing to the progression of SCA1. Disruption of the interaction between the polyQexpanded ATXN1 and CIC inhibited the SCA1 disease phenotypes in mice, suggesting that SCA1 is caused by neurotoxicity driven by a gain-of-function of the polyQexpanded ATXN1-CIC complex ${ }^{16}$.

\section{Cancer}

The first evidence for an association between CIC and cancer progression was the identification of the fusion between CIC and DUX4 as a result of a recurrent chromosomal translocation $\mathrm{t}(4 ; 19)(\mathrm{q} 35 ; \mathrm{q} 13)$ in Ewing-like 
sarcomas ${ }^{17}$. The CIC-DUX4 chimaeras are composed of the majority of the $\mathrm{CIC}$ protein, except for a small portion of the C-terminus, and the C-terminal region of DUX4 involved in transcriptional activation ${ }^{17}$. The CIC-DUX4 fusion protein acquires transforming activity against NIH3T3 fibroblasts, indicating that $C I C-D U X 4$ acts as a dominant oncogene ${ }^{17,39}$. The chimeric proteins transcriptionally activate the expression of CIC target genes, including PEA3 group genes that encode the oncogenic transcription factors ETV1, ETV4, and ETV $5^{17,18}$. Several other studies have identified various additional chromosomal translocations generating $C I C-D U X 4$ chimeric transcripts in round cell sarcoma as well as Ewing sarcoma $^{40-44}$. A xenograft mouse model subcutaneously injected with embryonic mesenchymal cells expressing CIC-DUX4 developed small round cell sarcoma ${ }^{45}$. Another study using a xenograft mouse model orthotopically injected with NIH3T3 mouse fibroblasts expressing CIC-DUX4 showed that the CIC-DUX4 proteins promote tumor growth and metastasis via the upregulation of CCNE1 and ETV4, respectively, suggesting that these proteins drive tumorigenesis and metastasis in sarcomas via distinct regulatory programs ${ }^{39}$.

CIC mutations occur most frequently in oligodendroglioma. Based on high-throughput DNA sequencing analyses, $C I C$ was shown to harbor point mutations in $50-70 \%$ of oligodendrogliomas carrying the codeletion of chromosomes $1 p$ and $19 q^{23,24,46}$. The role of CIC point mutations in oligodendroglioma development and progression has not been experimentally verified. However, CIC deficiency promoted gliomagenesis in a xenograft mouse model orthotopically injected with PDGFBexpressing neural stem cells (NSCs) ${ }^{27}$. The glial cellspecific deletion of $\mathrm{CIC}$ did not induce tumor formation in the mouse brain, suggesting that defects in CIC itself may not be sufficient to initiate oligodendroglioma ${ }^{25}$. Many somatic mutations in $C I C$, including truncations, insertions, and deletions, have been identified in advanced-stage human lung adenocarcinoma specimens ${ }^{22}$. Okimoto et al. showed that the inactivation of CIC by point mutations promotes lung cancer metastasis via derepression of ETV4, which induces the expression of MMP24 $4^{22}$. The CIC-ETV4-MMP24 metastatic axis is also involved in gastric adenocarcinoma ${ }^{22}$. Genetic ablation of CIC in adult mice caused $\mathrm{T}$ cell acute lymphoblastic leukemia/lymphoma (T-ALL) ${ }^{25,26}$, suggesting that CIC mutations could be considered driver mutations for $\mathrm{T}$ ALL in humans. T-ALL also developed in hematopoietic lineage cell-specific Cic null mice ${ }^{26}$. However, $\mathrm{T}$ cellspecific $\mathrm{Cic}$ null mice did not show T-ALL phenotypes up to 14 months of age ${ }^{33}$, suggesting that the loss of CIC in $\mathrm{T}$ cells may be insufficient to cause T-ALL, and CIC deficiency in other types of immune cells may contribute to disease onset in mice. Decreased CIC expression at the protein level is frequently observed in various types of cancer $^{19-21,28,46}$. Moreover, the CIC protein levels are often not correlated with the mRNA levels within the same cancer samples, suggesting that $\mathrm{CIC}$ exhibits robust post-transcriptional regulation in cancer cells ${ }^{19,21}$. Nuclear expression of CIC decreases gradually as prostate cancer (PC) becomes more aggressive ${ }^{20}$. CIC levels are also substantially downregulated in hepatocellular carcinoma (HCC), glioblastoma (GBM), and colorectal cancer $(\mathrm{CRC})^{19,21,28}$. The decreased expression of $\mathrm{CIC}$ leads to the derepression of $P E A 3$ group genes, thereby promoting cell growth and invasion in PC, HCC, GBM, and CRC cell lines ${ }^{19-21,28}$. Notably, the major PEA3 group members (e.g., ETV1, ETV4, or ETV5) regulated by CIC differ among cancer cell types; the expression of ETV5 and ETV4 is most highly and significantly upregulated by CIC deficiency in PC and HCC cell lines, respectively ${ }^{20,21}$. CIC is also involved in the control of cancer stem cell properties. CIC deficiency promotes the self-renewal capacity and increases the expression of cancer stem cell markers, including $\mathrm{EpCAM}^{+} / \mathrm{CD} 44^{\mathrm{hi}} / \mathrm{CD} 24^{\mathrm{lo}}$ and $\mathrm{ALDH}^{47,48}$, via derepression of ETV4, ETV5, and SOX2 in breast cancer cell lines ${ }^{49}$. Consistent with this result, CIC levels were decreased in breast cancer patient samples with a CD44 high and CD24 low phenotype ${ }^{49}$. These data suggest that CIC suppresses breast cancer formation by restricting cancer stemness and identify $\mathrm{CIC}$ as a potential regulator of stem cell maintenance.

\section{Functions of $\mathrm{CIC}$ in development Lung development}

Defective lung alveolarization has been observed in Cic- $\mathrm{L}^{-/-}$mice, in which CIC-L expression is completely abolished and CIC-S expression is substantially reduced but incompletely blocked ${ }^{15,31}$. Cic- $L^{-/-}$mice exhibited perinatal lethality; approximately $83 \%$ of $\mathrm{Cic}-\mathrm{L}^{-/-}$mice died before postnatal day 14 (P14; unpublished data), and the survivors were smaller than the wild-type (WT) littermates ${ }^{31}$. Cic- $L^{-/-}$survivors had lung alveolarization defects causing air space enlargement accompanied by MMP9 overexpression in the lungs at $\mathrm{P}^{31}$. Another germline $\mathrm{Cic}$ mutant $\left(\mathrm{Cic}^{\Delta 2-6 / \Delta 2-6}\right)$ mouse with deletions in Cic exons 2-6 (i.e., the HMG box-encoding exons), which expresses mutant CIC-L and CIC-S isoforms that lack the HMG box in the whole body, also exhibited defects in the terminal differentiation of the respiratory epithelium at embryonic day 18.5 (E18.5), potentially leading to delayed or altered alveolar maturation during postnatal development ${ }^{25}$. The CIC levels were relatively high in the lungs of E18.5 embryos ${ }^{31}$.

\section{Abdominal wall closure \\ Characterization of $\mathrm{Cic}^{\triangle 2-6 / \Delta 2-6}$ mice revealed that CIC is required for late embryonic development. Homozygous}


$\mathrm{Cic}^{\triangle 2-6 / \Delta 2-6}$ embryos were present in Mendelian ratios at E18.5 but died immediately after birth ${ }^{25}$. Approximately $70 \%$ of the E18.5 $\mathrm{Cic}^{\Delta 2-6 / \triangle 2-6}$ embryos had an omphalocele, a mild type of abdominal wall closure defect ${ }^{25}$. In this case, the gut protrudes into the umbilical ring in the late embryonic stage. Therefore, one explanation for the early death of Cic mutant mice is that a part of the internal organs, such as the intestines, is cannibalized when the mother removes the placenta after birth ${ }^{31,50}$. The abdominal wall closure defect was also found in mice that lack the expression of ATXN1 and ATXN1-like (ATNX1L), which bind to and stabilize $\mathrm{CIC}^{16,31,51}$ (Fig. 1b). Approximately $45 \%$ of the E18.5 Atxn1 and Atxn 1 l double null embryos had an omphalocele ${ }^{31}$. Taken together, these findings suggest that the CIC-ATXN1/ATXN1L complex is essential for normal embryogenesis and viability.

\section{Brain development and function}

CIC is highly expressed in the brain ${ }^{30,31}$. This molecule has been implicated in granule cell development based on the observation that $\mathrm{Cic}$ is highly expressed in immature granule cells in the cerebellum, hippocampus, and olfactory bulb ${ }^{12}$. A study of Cic mutant mice uncovered a critical role of CIC in brain development and function ${ }^{32}$. The deletion of Cic in the forebrain significantly reduced the thickness of cortical layers 2-4 and the dentate gyrus, potentially due to defects in the maintenance of postmitotic neurons ${ }^{32}$. The layer $2 / 3$ pyramidal neurons of the forebrain-specific $\mathrm{Cic}$ null $\left(\mathrm{Cic}^{f / f} ; \mathrm{Em} \times 1-\mathrm{Cre}\right)$ mice also had defective dendritic branching ${ }^{32}$. CIC deficiency in the forebrain caused learning and memory deficits, and a loss of $\mathrm{CIC}$ in the hypothalamus and medial amygdala led to defects in social interactions ${ }^{32}$. Consistent with these mouse data, de novo heterozygous truncating mutations in $C I C$ are associated with autism spectrum disorder, developmental delay/intellectual disability, seizures, and attention deficit hyperactivity disorder in humans ${ }^{32}$.

CIC is also associated with NSC maintenance and differentiation. Cic null NSCs presented EGF-independent hyperproliferative characteristics ${ }^{27}$. Hyperproliferation of NSCs by the loss of Cic was also confirmed in E13 embryos by a 5-ethynyl-2'-deoxyuridine (EdU) labeling experiment ${ }^{29}$. Upon the induction of differentiation in vitro, Cic null NSCs could not differentiate into mature oligodendrocytes and instead were maintained in an oligodendrocyte progenitor cell (OPC)-like stemness state ${ }^{27}$. A similar result was obtained using another forebrainspecific Cic null $\left(\mathrm{Cic}^{\mathrm{fff}}\right.$; Foxg1-Cre) mouse model, in which Olig2 ${ }^{+} \mathrm{Sox}^{+}{ }^{+}$cells and Olig2 ${ }^{+} \mathrm{Pdgfra}^{+}$OPCs are increased and $\mathrm{CNPase}^{+}$immature oligodendrocytes are decreased in the cortex ${ }^{29}$. Moreover, CIC deficiency enhanced the self-renewal capacity and promoted the symmetric division of $\mathrm{NSCs}^{29}$. Mechanistically, the derepression of Etv5 mediated the effects of CIC deficiency in $\mathrm{NSCs}^{29}$. Thus, $\mathrm{CIC}$ is a key transcription factor that controls brain development and function as well as the pathogenesis of neurological disorders.

\section{Immune cell development and function}

Park et al. investigated the role of CIC in the immune system by generating and characterizing hematopoietic lineage cell-specific Cic null $\left(\mathrm{Cic}^{f / f} ; \mathrm{Vav1-Cre}\right)$ mice $^{33}$. These mice had lymphoproliferative disorder-like symptoms at 9 weeks of age, as evidenced by an increased splenocyte count mainly due to the expansion of the $\mathrm{B}_{22} 2 \mathrm{O}^{+} \mathrm{B}$ cell population and hyperglobulinemia. $\mathrm{Cic}^{\mathrm{f} f \mathrm{f}}$; Vav1-Cre mice eventually developed systemic autoimmune-like phenotypes, including the enlargement of secondary lymphoid organs; increased anti-dsDNA antibody serum levels; immune cell infiltration into various organs, including the liver, lung, and kidney; and IgG deposition at the glomeruli of the kidney. $\mathrm{T}$ cell-specific $\mathrm{Cic}$ null $\left(\mathrm{Cic}^{\mathrm{fff}} ; \mathrm{Cd} 4-\mathrm{Cre}\right)$ mice also exhibited similar phenotypes to $\mathrm{Cic}^{f / f}$;Vav1-Cre mice, suggesting that CIC deficiency in $\mathrm{T}$ cells is critical for the induction of autoimmune-like symptoms ${ }^{33}$. CIC deficiency promotes the differentiation of follicular helper $\mathrm{T}$ (Tfh) cells ${ }^{33}$, which play a pivotal role in the germinal center reaction to produce isotype class switched high affinity antibodies against specific antigens ${ }^{52}$. At the molecular level, Etv5 is a critical target gene of CIC for the regulation of Tfh cell differentiation ${ }^{33}$. ETV5 levels were significantly upregulated in Cic null Tfh cells compared with WT cells. Adoptive transfer experiments using OT-II cells, ovalbumin-specific $\mathrm{T}$ cell receptor-expressing $\mathrm{CD} 4^{+}$ $\mathrm{T}$ cells, revealed that ETV5 overexpression promotes Tfh cell development and that the knockdown of ETV5 substantially rescues the enhanced Tfh cell differentiation of Cic null OT-II cells ${ }^{33}$. These results indicate that the CIC-ETV5 axis controls Tfh cell development. Park et al. also proposed that Maf, which encodes a transcription factor that promotes Tfh cell differentiation $^{53}$, is a target of ETV5 in CD4 ${ }^{+}$T cells under STAT3 activation $^{33}$.

$\mathrm{CIC}$ is also involved in maintaining homeostasis of bone marrow hematopoietic stem and progenitor cells (HSPCs) and early $\mathrm{T}$ cell development ${ }^{26}$. Analyses of bone marrow and thymic cells in adult stage-specific $\left(\mathrm{Cic}^{f f f} ; U B C\right.$-Cre/ $E R T 2$ ) and endothelial and hematopoietic lineage cellspecific $\left(\mathrm{Cic}^{\mathrm{fff}} ; \mathrm{Tek}-\mathrm{Cre}\right) \mathrm{Cic}$ null mice have shown that the number of HSPCs, including hematopoietic stem cells (HSCs) and multipotent progenitors (MPPs), is reduced, whereas the frequency of thymic double negative 1 (DN1) cells is significantly increased ${ }^{26}$. The frequency of early $\mathrm{T}$ cell precursors (ETPs), a subset of DN1 cells from the bone marrow that remain pluripotent, is also elevated in the thymus of $\mathrm{Cic}^{f / f} ; U B C$-Cre/ERT2 mice, suggesting that 
CIC regulates the self-renewal capacity of stem-like cells ${ }^{26}$.

CIC has been implicated in the development of $\mathrm{CD}^{+}$ resident memory $\mathrm{T}(\mathrm{Trm})$ cells in the liver ${ }^{34}$. Cic- $\mathrm{L}^{-/-}$ mice exhibit liver damage, as evidenced by increases in serum alanine transaminase (ALT) and hepatic proinflammatory cytokine expression levels ${ }^{30}$. These mice also have defects in the enterohepatic circulation of bile acids accompanied by the downregulation of several key genes involved in bile acid biosynthesis and transport in the liver $^{30}$. These liver dysfunctions are not due to a CIC deficiency in hepatocytes because liver-specific Cic null $\left(\mathrm{Cic}^{f / f} ; \mathrm{Alb}-\mathrm{Cr} e\right)$ mice do not recapitulate these phenotypes $^{34}$. Cic ${ }^{f f f} ; \mathrm{Cd}$-Cre mice have increased serum ALT and hepatic proinflammatory cytokine expression levels, indicating that $\mathrm{CIC}$-deficient $\mathrm{T}$ cells cause inflammatory liver injury ${ }^{34}$. CIC deficiency promotes the formation of liver $\mathrm{CD}^{+}$Trm-like cells expressing surface markers, such as $\mathrm{CD} 9^{+}, \mathrm{CD}_{49 \mathrm{a}^{+}}, \mathrm{CXCR6}^{+}, \mathrm{CXCR}^{+}$, and $\mathrm{CD} 103^{-}$, in a cell intrinsic manner ${ }^{34}$. Moreover, the suppression of liver $\mathrm{CD}^{+}$Trm-like cell formation dramatically mitigated liver injury phenotypes in $\mathrm{Cic}^{f / f} ; \mathrm{Cd} 4-\mathrm{Cre}$ mice treated with acetaminophen, which induces acute liver injury, suggesting that the increased $\mathrm{CD} 8^{+}$Trm-like cell population in the liver is responsible for the CIC deficiency-induced liver injury ${ }^{34}$. Mechanistically, the CIC-ETV5 axis controls liver $\mathrm{CD}^{+}{ }^{+}$Trm-like cell differentiation. The derepression of ETV5 induces the expression of HOBIT, a transcription factor required for Trm cell development ${ }^{54}$, in Cic null CD8 ${ }^{+} \mathrm{T}$ cells, thereby promoting Trm cell differentiation ${ }^{34}$.

\section{Regulation of CIC}

RTK-RAS-MAPK pathways suppress CIC activity via the cytoplasmic translocation and/or degradation of CIC (Fig. 1b). This regulatory mechanism was originally discovered in studies of CIC expression patterns in Drosophila embryos. Torso RTK signaling in the early embryo leads to the degradation of CIC, whereas EGFR signaling in the ovarian follicle induces the partial relocalization of $\mathrm{CIC}$ to the cytoplasm ${ }^{10,55}$. EGF treatment resulted in the phosphorylation of human CIC-S at 20 different serine/ threonine residues, presumably by ERK and $\mathrm{p} 90^{\mathrm{RSK}}$, a kinase activated by ERK ${ }^{56}$. In particular, $\mathrm{p} 90^{\mathrm{RSK}}$-mediated phosphorylation of S173 is critical for 14-3-3 binding (Fig. 1a), which inhibits CIC binding to target DNA sequences ${ }^{56}$. S1409 phosphorylation prevents the binding of importin $\alpha 4 /$ KPNA3 to the nuclear localization signal of $\mathrm{CIC}^{56}$. However, the disruption of the CIC-KPNA3 interaction does not affect the nuclear localization of CIC-S ${ }^{56}$, suggesting that other transport-related factors might be required for the cytoplasmic translocation of CIC in mammalian cells. ERK binds to the C-terminal region of human CIC-S containing residues 1335-1359 (prior to the $\mathrm{C} 1$ domain; Fig. 1a) ${ }^{57}$. EGFR stimulation decreased CIC levels in mammalian cells ${ }^{15,19,22}$. The inhibition of ERK by treatment with MEK1/2 inhibitors increased the levels of nuclear CIC-S at the expense of cytoplasmic CIC expression in pancreatic cancer cells ${ }^{58}$, suggesting that ERK regulates the subcellular localization of CIC (Fig. 1b). Moreover, EGFR-activated c-Src tyrosine kinase mediates cytoplasmic translocation of CIC-S via phosphorylation of the Y1455 residue ${ }^{59}$ (Fig. 1a, b). CIC is degraded in the nucleus upon EGFR-ERK activation ${ }^{19}$. In this process, the nuclear E3 ligase PRAJA1 (PJA1) polyubiquitylates $\mathrm{CIC}$, leading to the proteasomal degradation of CIC in the nucleus ${ }^{19}$ (Fig. 1b). DNA binding of CIC is a prerequisite for the PJA1-mediated polyubiquitylation of $\mathrm{CIC}^{19}$. In addition, PJA1 recognizes the S173 residue of CIC-S to interact with $\mathrm{CIC}^{19}$. Since 14-3-3 also binds to S173-phosphorylated CIC-S to control the transcriptional repressor activity of $\mathrm{CIC}^{56}$, crosstalk between 14-3-3 and PJA1 might be involved in the regulation of CIC activity and/or stability.

Another regulatory mechanism underlying $\mathrm{CIC}$ activity is the ATXN1/ATXN1L interaction-mediated stabilization of CIC (Fig. 1b). Both ATXN1 and its homolog ATXN1L interact with and stabilize $\mathrm{CIC}^{31}$. The AXH domain of ATXN1/ATXN1L and the highly conserved Nterminal region of CIC-S, including amino acid residues 28-48, mediate their interaction ${ }^{60}$ (Fig. 1a). ATXN1L plays a more pivotal role in the stabilization of CIC than ATXN1; CIC levels decreased more substantially in response to the loss of ATXN1L than to the loss of ATXN1, leading to substantial derepression of CIC target gene expression $^{31,61}$. In the absence of ATXN1L, CIC becomes unstable, resulting in proteasomal degradation ${ }^{61}$. ATXN1L also promotes CIC binding to the target gene promoter regions ${ }^{61}$. However, the reason for the relative importance of ATXN1L for CIC stabilization and function is unclear.

Long noncoding RNA (lncRNA)-mediated regulation of $C I C$ expression has been reported ${ }^{62}$. The levels of $C I C$ and IncRNA-AC006129.1, of which genomic locus is close to $C I C$ in chromosome 19 , were significantly decreased and increased in samples from schizophrenia patients, respectively $^{62}$. AC006129.1 transgenic mice exhibited social interaction deficits, spatial working memory impairments, and sensorimotor gating disruption accompanied by upregulation of inflammatory response genes, including SOCS3 and CASP1, which are CIC target genes $^{62}$. The overexpression of AC006129.1 downregulated CIC levels in both mouse and human cells ${ }^{62}$, suggesting that this IncRNA-mediated transcriptional repression of CIC expression might be conserved in mammals. Mechanistically, AC006129.1 recruits DNA methyltransferases 1 and 3a (DNMT1 and DNMT3a) and induces DNA methylation of CIC promoter regions ${ }^{62}$. 
The AC006129.1-mediated suppression of CIC expression leads to derepression of SOCS3 and CASP1, potentially contributing to the pathogenesis of schizophrenia ${ }^{62}$.

\section{Concluding remarks}

$\mathrm{CIC}$ has multiple roles in various developmental processes and in the pathogenesis of various diseases. CIC is believed to function as a tumor suppressor in various types of cancer and is a regulator of embryogenesis, brain and immune cell development, and stem cell maintenance. Our current understanding of CIC functions in mammals is largely limited to processes regulated by the CIC-ETV1/ETV4/ETV5 axis. Many molecular studies of mammalian cells have identified additional target genes of CIC, such as Spry4, Dusp4, Dusp6, Spred1, Ccnd1, Ccne1, and Per $2^{15,17,27,39,63,64}$. It will be important to clarify the effects of CIC regulation of various target genes at both the cellular and organismal levels. Furthermore, the mechanism by which $\mathrm{CIC}$ regulates target gene expression remains largely unclear and should be a focus of future research. CIC was shown to recruit the histone deacetylase complex to repress the expression of target genes in stem cells ${ }^{64}$. Another study proposed that CIC has dual functions as a transcriptional activator as well as a repressor $^{27}$. There are several unanswered questions regarding the regulation of CIC activity. For example, which factors mediate the cytoplasmic translocation of CIC upon the activation of RTK signaling? How does ATXN1L stabilize CIC at the molecular level? Which transcription factors control the expression of CIC? These unresolved issues need to be addressed for a comprehensive understanding of the CIC-mediated regulation of biological processes. Finally, CIC is emerging as a key determinant of immune responses. A few studies have recently uncovered the roles of $\mathrm{CIC}$ in the development of $\mathrm{T}$ cell subsets ${ }^{26,33,34}$. However, the function of $\mathrm{CIC}$ in other types of immune cells, including B cells, dendritic cells, and macrophages, has not been established. Comprehensive studies of CIC functions in various types of immune cells will improve our understanding of the pathogenesis of immune disorders, such as autoimmune diseases and lymphomas, at the molecular level.

\section{Acknowledgements}

This work was supported by grants from the National Research Foundation (NRF) of Korea (2017R1A5A1015366 and 2018R1A2B2004416).

Conflict of interest

The author declars no conflict of interest.

\section{Publisher's note}

Springer Nature remains neutral with regard to jurisdictional claims in published maps and institutional affiliations.
Received: 23 January 2020 Revised: 27 February 2020 Accepted: 3 March 2020.

Published online: 1 April 2020

\section{References}

1. Jimenez, G., Guichet, A., Ephrussi, A. \& Casanova, J. Relief of gene repression by torso RTK signaling: role of capicua in Drosophila terminal and dorsoventral patterning. Genes Dev. 14, 224-231 (2000).

2. Andreu, M. J. et al. EGFR-dependent downregulation of Capicua and the establishment of Drosophila dorsoventral polarity. Fly 6, 234-239 (2012).

3. Andreu, M. J. et al. Mirror represses pipe expression in follicle cells to initiate dorsoventral axis formation in Drosophila. Development 139, 1110-1114 (2012).

4. Atkey, M. R., Lachance, J. F., Walczak, M., Rebello, T. \& Nilson, L. A. Capicua regulates follicle cell fate in the Drosophila ovary through repression of mirror. Development 133, 2115-2123 (2006).

5. Cinnamon, E. et al. Capicua integrates input from two maternal systems in Drosophila terminal patterning. EMBO J. 23, 4571-4582 (2004).

6. Goff, D. J., Nilson, L. A. \& Morisato, D. Establishment of dorsal-ventral polarity of the Drosophila egg requires capicua action in ovarian follicle cells. Development 128, 4553-4562 (2001).

7. Herranz, H., Hong, X. \& Cohen, S. M. Mutual repression by bantam miRNA and Capicua links the EGFR/MAPK and Hippo pathways in growth control. Curr. Biol. 22, 651-657 (2012)

8. Roch, F., Jimenez, G. \& Casanova, J. EGFR signalling inhibits Capicuadependent repression during specification of Drosophila wing veins. Development 129, 993-1002 (2002).

9. Yang, L. et al. Minibrain and Wings apart control organ growth and tissue patterning through down-regulation of Capicua. Proc. Natl Acad. Sci. USA 113, 10583-10588 (2016).

10. Astigarraga, S. et al. A MAPK docking site is critical for downregulation of Capicua by Torso and EGFR RTK signaling. EMBO J. 26, 668-677 (2007).

11. Jimenez, G., Shvartsman, S. Y. \& Paroush, Z. The Capicua repressor-a general sensor of RTK signaling in development and disease. J. Cell Sci. 125, 1383-1391 (2012).

12. Lee, C. J. et al. CIC, a member of a novel subfamily of the HMG-box superfamily, is transiently expressed in developing granule neurons. Mol. Brain Res. 106, 151-156 (2002).

13. Fores, M. et al. A new mode of DNA binding distinguishes Capicua from other HMG-box factors and explains its mutation patterns in cancer. PLoS Genet. 13, e1006622 (2017).

14. Lam, Y. C. et al. ATAXIN-1 interacts with the repressor Capicua in its native complex to cause SCA1 neuropathology. Cell 127, 1335-1347 (2006).

15. Fryer, J. D. et al. Exercise and genetic rescue of SCA1 via the transcriptional repressor Capicua. Science 334, 690-693 (2011).

16. Rousseaux, M. W. C. et al. ATXN1-CIC complex is the primary driver of cerebellar pathology in spinocerebellar Ataxia Type 1 through a gain-of-function mechanism. Neuron 97, 1235-1243 (2018).

17. Kawamura-Saito, M. et al. Fusion between CIC and DUX4 up-regulates PEA3 family genes in Ewing-like sarcomas with $\mathrm{t}(4 ; 19)(\mathrm{q} 35 ; \mathrm{q} 13)$ translocation. Hum. Mol. Genet. 15, 2125-2137 (2006).

18. Oh, S., Shin, S. \& Janknecht, R. ETV1, 4 and 5: an oncogenic subfamily of ETS transcription factors. Biochim. Biophys. Acta 1826, 1-12 (2012).

19. Bunda, S. et al. CIC protein instability contributes to tumorigenesis in glioblastoma. Nat. Commun. 10, 661 (2019)

20. Choi, N. et al. miR-93/miR-106b/miR-375-CIC-CRABP1: a novel regulatory axis in prostate cancer progression. Oncotarget 6, 23533-23547 (2015).

21. Kim, E. et al. Capicua suppresses hepatocellular carcinoma progression by controlling the ETV4-MMP1 axis. Hepatology 67, 2287-2301 (2018).

22. Okimoto, R. A. et al. Inactivation of Capicua drives cancer metastasis. Nat. Genet. 49, 87-96 (2017).

23. Bettegowda, C. et al. Mutations in CIC and FUBP1 contribute to human oligodendroglioma. Science 333, 1453-1455 (2011).

24. Chan, A. K. et al. Loss of CIC and FUBP1 expressions are potential markers of shorter time to recurrence in oligodendroglial tumors. Mod. Pathol. 27, 332-342 (2014).

25. Simon-Carrasco, L. et al. Inactivation of Capicua in adult mice causes T-cell lymphoblastic lymphoma. Genes Dev. 31, 1456-1468 (2017). 
26. Tan, Q. et al. Loss of Capicua alters early T cell development and predisposes mice to T cell lymphoblastic leukemia/lymphoma. Proc. Natl Acad. Sci. USA 115, E1511-E1519 (2018).

27. Yang, R. et al. Cic loss promotes gliomagenesis via aberrant neural stem cell proliferation and differentiation. Cancer Res. 77, 6097-6108 (2017).

28. Lee, J. S. et al. Capicua suppresses colorectal cancer progression via repression of ETV4 expression. Cancer Cell Int. 20, 42 (2020).

29. Ahmad, S. T. et al. Capicua regulates neural stem cell proliferation and lineage specification through control of Ets factors. Nat. Commun. 10, 2000 (2019).

30. Kim, E. et al. Deficiency of Capicua disrupts bile acid homeostasis. Sci. Rep. 5, 8272 (2015).

31. Lee, $Y$. et al. ATXN1 protein family and CIC regulate extracellular matrix remodeling and lung alveolarization. Dev. Cell 21, 746-757 (2011).

32. Lu, H. C. et al. Disruption of the ATXN1-CIC complex causes a spectrum of neurobehavioral phenotypes in mice and humans. Nat. Genet. 49, 527-536 (2017).

33. Park, S. et al. Capicua deficiency induces autoimmunity and promotes follicular helper T cell differentiation via derepression of ETV5. Nat. Commun. 8, 16037 (2017).

34. Park, S., Park, J., Kim, E. \& Lee, Y. The Capicua/ETS translocation variant 5 axis regulates liver-resident memory $\mathrm{CD} 8(+)$ T-cell development and the pathogenesis of liver injury. Hepatology 70, 358-371 (2019).

35. Kang, S. \& Hong, S. Molecular pathogenesis of spinocerebellar ataxia type 1 disease. Mol. Cells 27, 621-627 (2009).

36. Orr, H. T. \& Zoghbi, H. Y. Trinucleotide repeat disorders. Annu. Rev. Neurosci. 30, 575-621 (2007).

37. Emamian, E. S. et al. Serine 776 of ataxin-1 is critical for polyglutamine-induced disease in SCA1 transgenic mice. Neuron 38, 375-387 (2003).

38. Perez Ortiz, J. M. \& Orr, H. T. Spinocerebellar Ataxia type 1: molecular mechanisms of neurodegeneration and preclinical studies. Adv. Exp. Med. Biol. 1049, 135-145 (2018).

39. Okimoto, R. A. et al. CIC-DUX4 oncoprotein drives sarcoma metastasis and tumorigenesis via distinct regulatory programs. J. Clin. Invest. 129, 3401-3406 (2019).

40. Graham, C., Chilton-MacNeill, S., Zielenska, M. \& Somers, G. R. The CIC-DUX4 fusion transcript is present in a subgroup of pediatric primitive round cell sarcomas. Hum. Pathol. 43, 180-189 (2012).

41. Italiano, A. et al. High prevalence of CIC fusion with double-homeobox (DUX4) transcription factors in EWSR1-negative undifferentiated small blue round cel sarcomas. Genes Chromosomes Cancer 51, 207-218 (2012).

42. Kajtar, B. et al. CD99-positive undifferentiated round cell sarcoma diagnosed on fine needle aspiration cytology, later found to harbour a CIC-DUX4 translocation: a recently described entity. Cytopathology 25, 129-132 (2014).

43. Mangray, S. et al. Primary undifferentiated sarcoma of the kidney harboring a novel variant of CIC-DUX4 gene fusion. Am. J. Surg. Pathol. 40, 1298-1301 (2016).

44. Tsukamoto, Y. et al. Primary undifferentiated small round cell sarcoma of the deep abdominal wall with a novel variant of t(10;19) CIC-DUX4 gene fusion. Pathol. Res Pract. 213, 1315-1321 (2017).

45. Yoshimoto, T. et al. CIC-DUX4 induces small round cell sarcomas distinct from Ewing sarcoma. Cancer Res. 77, 2927-2937 (2017).

46. Gleize, V. et al. ClC inactivating mutations identify aggressive subset of 1 19 19 codeleted gliomas. Ann. Neurol. 78, 355-374 (2015).
47. Ginestier, $\mathrm{C}$. et al. ALDH1 is a marker of normal and malignant human mammary stem cells and a predictor of poor clinical outcome. Cell Stem Cell $\mathbf{1}$ 555-567 (2007).

48. Ponti, D., Zaffaroni, N., Capelli, C. \& Daidone, M. G. Breast cancer stem cells: an overview. Eur. J. Cancer 42, 1219-1224 (2006).

49. Yoe, J., Kim, D., Kim, S. \& Lee, Y. Capicua restricts cancer stem cell-like properties in breast cancer cells. Oncogene https://doi.org/10.1038/s41388-020-1230-7 (2020).

50. Thumkeo, D., Shimizu, Y., Sakamoto, S., Yamada, S. \& Narumiya, S. ROCK-I and ROCK-II cooperatively regulate closure of eyelid and ventral body wall in mouse embryo. Genes Cells 10, 825-834 (2005).

51. Bowman, A. B. et al. Duplication of Atxn1l suppresses SCA1 neuropathology by decreasing incorporation of polyglutamine-expanded ataxin-1 into native complexes. Nat. Genet. 39, 373-379 (2007).

52. Crotty, S. Follicular helper CD4 T cells (TFH). Annu. Rev. Immunol. 29, 621-663 (2011).

53. Bauquet, A. T. et al. The costimulatory molecule ICOS regulates the expression of C-Maf and IL-21 in the development of follicular T helper cells and $\mathrm{TH}-17$ cells. Nat. Immunol. 10, 167-175 (2009).

54. Mackay, L. K. et al. Hobit and Blimp1 instruct a universal transcriptional program of tissue residency in lymphocytes. Science 352, 459-463 (2016).

55. Ajuria, L. et al. Capicua DNA-binding sites are general response elements for RTK signaling in Drosophila. Development 138, 915-924 (2011).

56. Dissanayake, $K$. et al. ERK/p90(RSK)/14-3-3 signalling has an impact on expression of PEA3 Ets transcription factors via the transcriptional repressor capicua. Biochem. J. 433, 515-525 (2011).

57. Futran, A. S., Kyin, S., Shvartsman, S. Y. \& Link, A. J. Mapping the binding interface of ERK and transcriptional repressor Capicua using photocrosslinking. Proc. Natl Acad. Sci. USA 112, 8590-8595 (2015).

58. Wang, B. et al. ATXN1L, CIC, and ETS transcription factors modulate sensitivity to MAPK pathway inhibition. Cell Rep. 18, 1543-1557 (2017).

59. Bunda, S. et al. c-Src phosphorylates and inhibits the function of the $\mathrm{CIC}$ tumor suppressor protein. Mol. Cancer Res. https://doi.org/10.1158/1541-7786.MCR18-1370 (2020).

60. Kim, E., Lu, H. C., Zoghbi, H. Y. \& Song, J. J. Structural basis of protein complex formation and reconfiguration by polyglutamine disease protein Ataxin-1 and Capicua. Genes Dev. 27, 590-595 (2013).

61. Wong, D. et al. Transcriptomic analysis of CIC and ATXN1L reveal a functional relationship exploited by cancer. Oncogene 38, 273-290 (2019).

62. Ni, C. et al. LnCRNA-AC006129.1 reactivates a SOCS3-mediated anti-inflammatory response through DNA methylation-mediated $\mathrm{CIC}$ downregulation in schizophrenia. Mol Psychiatry https://doi.org/10.1038/s41380-020-0662-3 (2020).

63. Crespo-Barreto, J., Fryer, J. D., Shaw, C. A., Orr, H. T. \& Zoghbi, H. Y. Partial loss of ataxin-1 function contributes to transcriptional dysregulation in spinocerebellar ataxia type 1 pathogenesis. PLoS Genet. 6, e1001021 (2010).

64. Weissmann, S. et al. The tumor suppressor CIC directly regulates MAPK pathway genes via histone deacetylation. Cancer Res. 78, 4114-4125 (2018) 